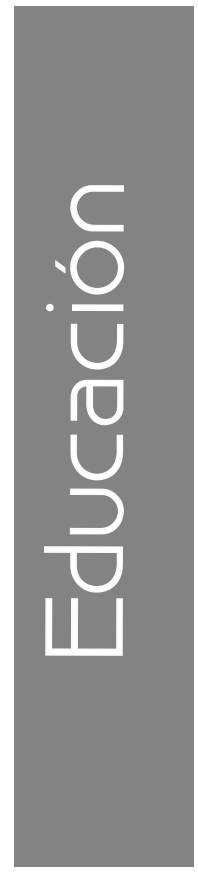

\title{
VISUAL BASIC PARA EXCEL EN LA ENSEÑANZA DE LA INGENIERÍA
}

\author{
Carlos Arturo Coronado R. * \\ José Isidro García**
}

*ngeniero Civil - Profesor de la Pontificia Universidad Javeriana de Cali

**Ingeniero Mecánico - Profesor de la Escuela de Ingeniería Mecánica de la Facultad de Ingeniería Universidad del Valle.

\section{RESUMEN}

Este artículo describe la importancia del uso de Visual Basic para Excel en la enseñanza de ingeniería. En él se presentan los conceptos básicos de Visual Basic y una aplicación específica en geotecnia, resaltando los alcances logrados en el proceso de aprendizaje cuando esta metodología es utilizada.

\section{ABSTRACT}

This paper describes the importance of using Visual Basic for Excel in engineering teaching. It presents the Visual basic fundamental concepts and a specific application in geotechnics, 
highlighting the achievements in learning processes when this methodology is used.

\section{INTRODUCCIÓN}

La computadora se convierte en una herramienta cada vez más necesaria en la práctica de la mayoría de las profesiones y más concretamente en la solución de problemas de ingeniería como geotecnia en Ingeniería Civil y diseño de elementos de máquinas en Ingeniería Mecánica. Sin embargo, por múltiples razones en las universidades aún no se ha intensificado su uso para la solución de problemas prácticos, dejando de lado la posibilidad de profundizar en los temas tratados a través del desarrollo de códigos, modelación y análisis paramétrico. Esta tarea generalmente se le asigna al estudiante de forma implícita, quien en la mayoría de los casos no la cumple, o incluso desconoce la posibilidad de realizarla. Por otro lado, los cursos de algoritmia y programación forman parte del nivel de formación básica de los programas académicos, lo cual hace que el estudiante no tenga la posibilidad de experimentar y desarrollar aplicaciones prácticas de su disciplina, ocasionándole la pérdida del ánimo y malos entendidos.

Adicionalmente, la enseñanza que usualmente se imparte de $C$ como lenguaje de programación y DOS como entorno de desarrollo, puede ocasionarle al estudiante poca aceptación por el uso del computador. Por el contrario, un entorno de programación como Visual Basic para Excel facilita el desarrollo rápido de aplicaciones con una interfaz gráfica aceptable, un lenguaje estructurado y la potencia de Excel para el uso de funciones de hoja de cálculo y gráficos. Por otro lado, si se tiene en cuenta la amplia difusión, aceptación y uso extensivo de Excel en las universidades y el medio, el uso de este entorno de desarrollo resulta recomendable para las aplicaciones típicas de la mayoría de los cursos de ingeniería.

Teniendo en cuenta las consideraciones anteriores, a continuación se presenta la experiencia de los autores en la enseñanza y aplicación de Visual Basic para Excel en diferentes cursos de geotecnia como primer curso evaluado.

\section{CONCEPTOS BÁSICOS DE VISUAL BASIC}

Visual Basic es uno de los lenguajes de programación de computadoras personales más usado entre los programadores expertos y principiantes. En el caso de los programadores expertos por la facilidad que brinda para el desarrollo de aplicaciones complejas en poco tiempo (comparado con el tiempo que toma programar en otros lenguajes visuales). En el caso de los principiantes por la facilidad de aprendizaje. Visual Basic para aplicaciones, permite combinar las facilidades de programación de Visual Basic con la potencia de Excel. De esta manera, se puede contar con las hojas de cálculo, gráficos y funciones incorporadas de dicha aplicación.

Se debe notar que Visual Basic es un lenguaje visual, también llamado de $4^{a}$ generación. Por lo tanto, gran cantidad de tareas se realizan sin escribir códigos, simplemente con operaciones gráficas realizadas con el ratón sobre la pantalla. Visual Basic es también un lenguaje basado en objetos, aunque no orientado a objetos. La diferencia está en que Visual Basic utiliza objetos con propiedades y métodos, pero carece de los mecanismos de herencia y polimorfismo propios de los verdaderos lenguajes orientados a objetos. Los programas desarrollados en Visual Basic, en general, son orientados a objetos manejados por eventos, esto facilita la interacción entre el usuario y la aplicación. De esta manera, una aplicación típica de Excel presenta una o más pantallas llenas de objetos tales como: cuadros de texto; botones de opción, de comando y de selección; listas e imágenes. Por otro lado, el usuario interactúa con estos objetos a través de eventos tales como el clic, lo cual, en general, da 
origen a una respuesta de la aplicación, dándose una nueva interacción y así sucesivamente.

Finalmente se debe mencionar que, para desarrollar una aplicación de Visual Basic para Excel, primero se diseña la interfaz de ingreso de datos. Luego, en el editor de Visual Basic se escribe el código que realizará las diferentes tareas. Por último, a través de Excel se enlazan el código desarrollado y los objetos específicos de la interfaz de usuario.

\section{MOTIVACIÓN}

Para la formación de los estudiantes de ingeniería de la Pontificia Universidad Javeriana - Cali y de la Universidad del Valle, se ha considerado necesario fomentar el manejo de herramientas computacionales. Ésto no se debe entender como el simple uso de programas comerciales, los cuales en muchos casos se emplean como "cajas negras" reduciendo al usuario a un simple apéndice del computador. Por lo tanto, resulta indispensable que el estudiante adquiera una actitud crítica, escéptica e incluso de desconfianza respecto a los resultados que un programa pueda generar. En este sentido, se ha notado que al promover el desarrollo de software el estudiante se acerca a la actitud mencionada.

Cuando el estudiante desarrolla el software para la solución de un problema de ingeniería se enfrenta a un proceso que implica los siguientes pasos:

1. Desarrollo de la interfaz de ingreso de datos o preproceso; en este punto se debe preocupar por la manera de comunicarse con el usuario y lograr que el proceso resulte limpio, sencillo y eficiente, lo cual motiva soluciones creativas y novedosas.

2. Algoritmo y codificación o solución; este paso, en general, implica un proceso de investigación y familiarización con el problema. Por otro lado, dado que el algoritmo debe resultar suficientemente robusto y general, es necesario que el estudiante imagine o genere diferentes situaciones hipotéticas, que puedan ser manejadas por el código. Es así como deberá investigar, crear e imaginar para dar solución al problema planteado.

3. Presentación de resultados o post-proceso; una vez solucionado el problema, el estudiante debe presentar los resultados de sus cálculos de una forma que permita su rápida asimilación y o interpretación. En este sentido el uso de gráficas de las magnitudes de interés prueba ser de gran utilidad, lo cual nuevamente implica un proceso altamente creativo.

4. Validación de resultados; finalmente resulta necesario confrontar los resultados obtenidos con soluciones analíticas y publicaciones reconocidas. Esto permite al estudiante desarrollar una actitud crítica y escéptica frente a sus programas y los de otros.

Como resultado final se notará que en la medida que una aplicación sea más complicada y aumenten las líneas de código fuente, lo más probable es que inicialmente el programa no haga lo que se desea, aún cuando se haya tenido mucho cuidado en su diseño o planeación. Parece que no importa qué tanto se esmere en hacer un programa robusto y libre de errores, siempre habrá alguien que, de alguna manera, encontrará la forma de hacerlo fallar; los programas comerciales no resultan ajenos a esta dolencia.

\section{EJEMPLO DE UNA APLICACIÓN DESARROLLADA DURANTE UN CURSO}

Como ya se dijo, el desarrollo de software fomenta el pensamiento creativo, la investigación y una actitud crítica y ordenada para la solución de problemas de ingeniería. Por otro lado, reduce la dependencia de software de terceros para la solución de problemas particulares. Además, permite profundizar en los temas tratados a través de la modelación y análisis paramétrico, labores indispensables con el fin de ganar 
sensibilidad respecto a un problema particular. Teniendo en cuenta los aspectos anteriores, los autores han promovido y desarrollado en sus diferentes cursos las aplicaciones que a continuación se mencionan.

\section{Mecánica de Suelos}

Durante estos cursos se ha promovido el desarrollo de software para clasificación de suelos, cálculo de esfuerzos, modelación de flujo de agua subterránea, solución de la ecuación de consolidación por series de Fourier y cálculo de asentamientos. En las siguientes líneas se presentan algunos ejemplos de las aplicaciones desarrolladas y sus usos.

\section{Esfuerzos debidos a cargas rectangulares}

El propósito de esta aplicación es ilustrar los principios de superposición y de Saint Venant, ampliamente usados en mecánica de suelos y sólidos. El principio de superposición es una consecuencia de la naturaleza lineal de la teoría de la elasticidad. Por otro lado el principio de Saint Venant establece que mientras las diferentes aproximaciones de un sistema de cargas sean estáticamente equivalentes, las soluciones resultantes serán válidas en regiones suficientemente alejadas del apoyo o del punto aplicación de la carga. Es decir, las soluciones pueden diferir en forma significativa sólo en la vecindad inmediata del soporte o punto de aplicación.

En este contexto se hace uso de estos principios para la solución del problema planteado de la siguiente manera: se divide la carga rectangular en una serie de cargas puntuales estáticamente equivalentes (principio de Saint Venant). Luego se usa el principio de superposición para calcular el esfuerzo en un punto como la sumatoria de los esfuerzos individuales producidos por cada carga puntual. En la Figura 1 se puede apreciar la división de la carga rectangular en la correspondiente serie de cargas puntuales equivalentes.

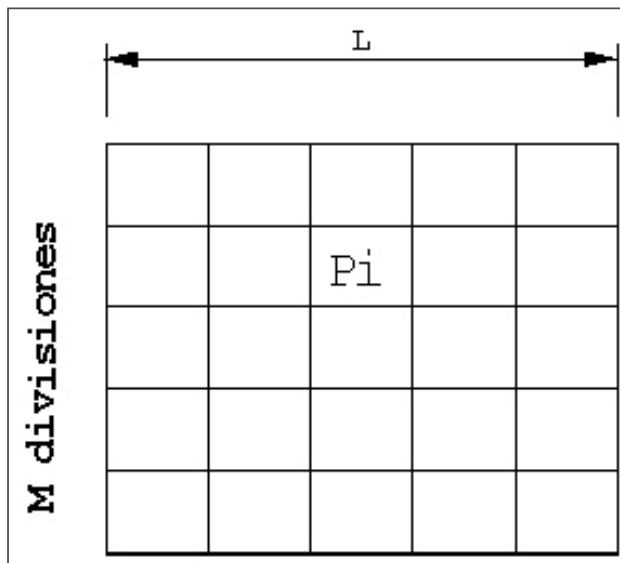

Dado que $q$ es la carga actuante por la unidad de superficie, la carga puntual equivalente $P i$, se puede determinar de la siguiente forma:

$$
P i=\frac{q L B}{N M}
$$

Donde:

\section{$L$ y $B$ son las dimensiones de la zapata}

$N M$ es el número de divisiones o cargas puntuales

Figura 1: División carga rectangular en cargas

puntuales equivalentes

Para el desarrollo de la presente aplicación se usa una hoja de cálculo, denominada Datos, donde se lee la información ingresada por el usuario y se presenta el resultado. En la Figura 2 se puede apreciar la apariencia general de la hoja de ingreso de datos.

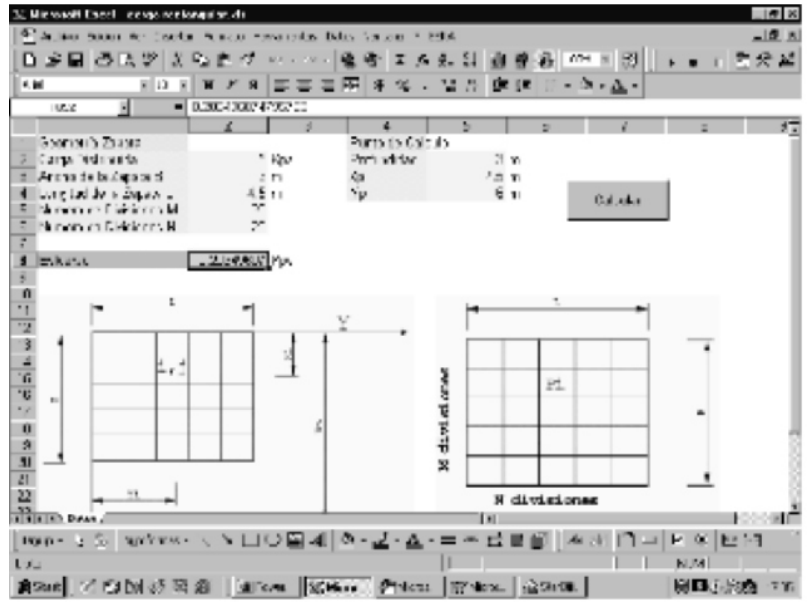

Figura 2: Apariencia de la hoja de ingreso de datos 
En la Figura 3 se indica la influencia del número de divisiones en la calidad de los resultados obtenidos, para el ejemplo 8.3 del libro de Lambe $\&$ Whitman. En la Figura 3b se calcula el esfuerzo en un punto más cerca de la carga aplicada; nótese que para una sola división el error es de aproximadamente el $65 \%$. Sin embargo, a partir de 5 divisiones el error es inferior al 1\%. De esta manera el estudiante puede llegar a sus propias conclusiones y valorar de mejor manera el significado de los principios anteriormente mencionados.

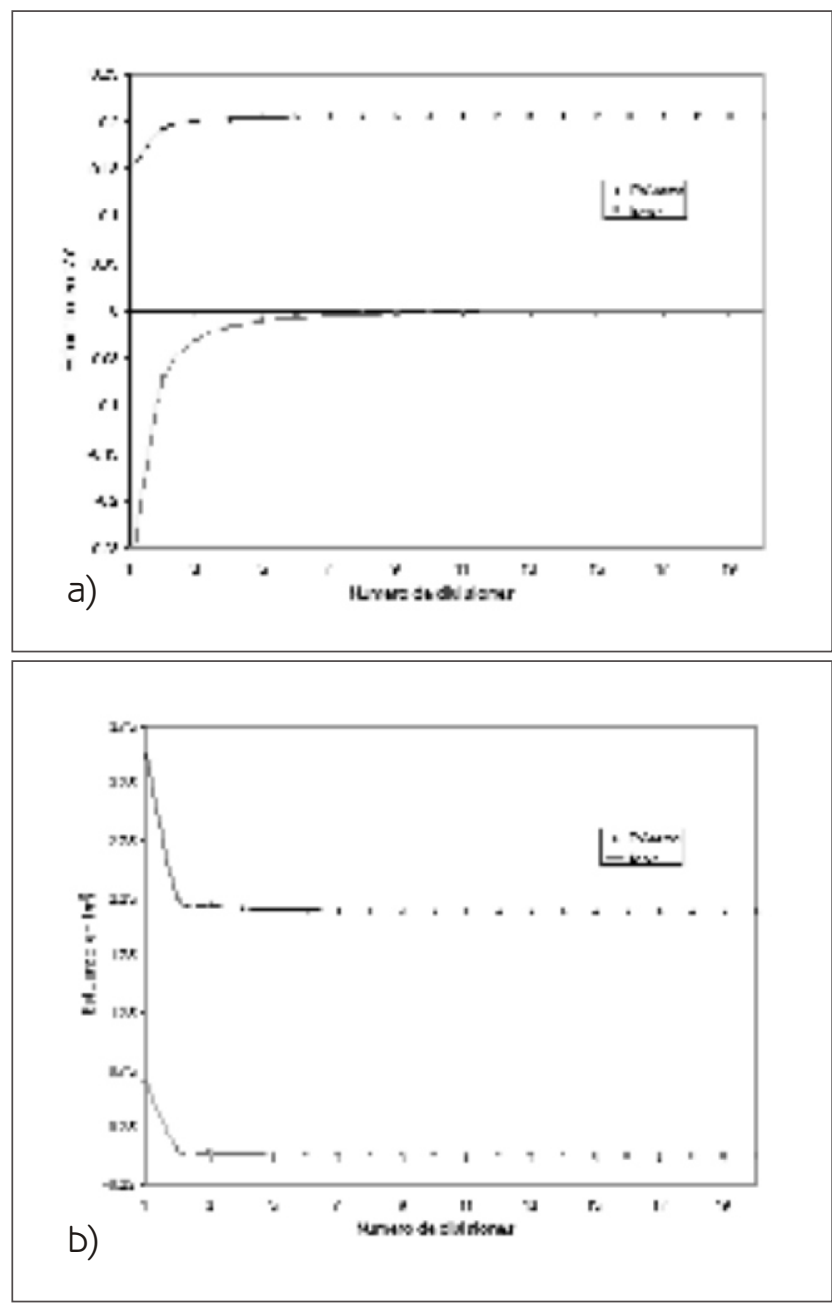

Figura 3. Influencia del número de divisiones

Cálculo de esfuerzos y deformaciones

El propósito de esta aplicación es ilustrar el uso del principio de superposición y la influencia de la geometría de una cimentación en la magnitud del esfuerzo y deformación.

La aplicación permite calcular el esfuerzo y deformación bajo un conjunto de cargas rectangulares (zapatas). Se debe notar que, desde el punto de vista práctico, interesa realizar el cálculo del esfuerzo bajo el centro de cada una de las zapatas y a diferentes profundidades de la masa de suelo.

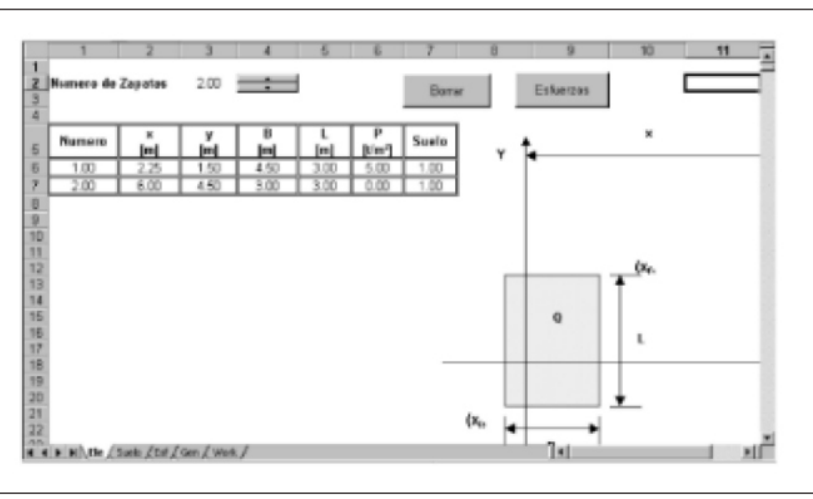

Figura 4. Hoja de Ingreso de cargas rectangulares

Para ilustrar el uso de la aplicación, a continuación se supone que desea calcular el esfuerzo bajo el centro de cada una de las zapatas de la cimentación que se indica en la Figura 5.

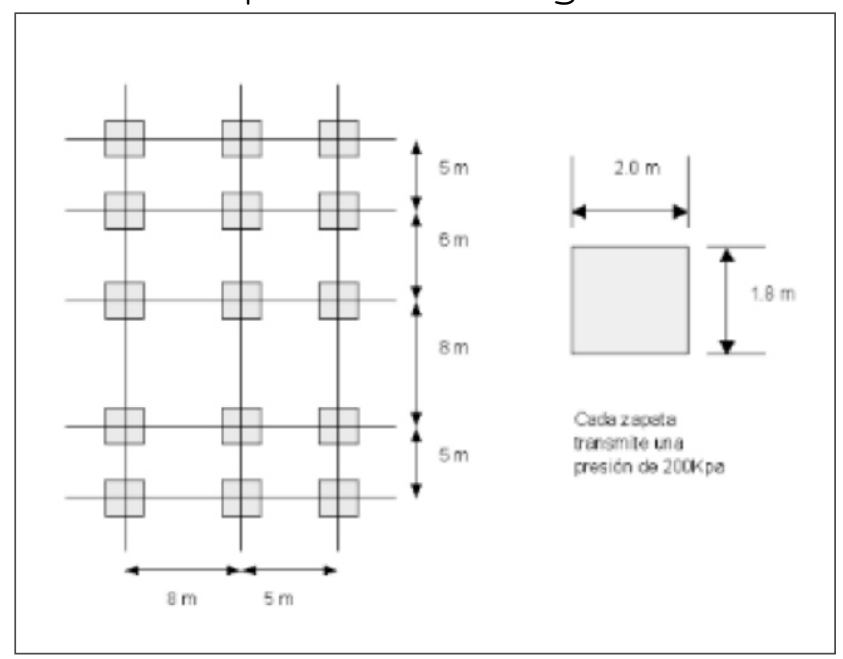

Figura 5. Geometría del problema

En la Figura 6 se indican los diferentes esfuerzos por eje de zapatas, para una profundidad de 
$7.5 \mathrm{~m}$. De esta manera se puede apreciar que las zapatas y ejes centrales experimentan los mayores incrementos de esfuerzo.

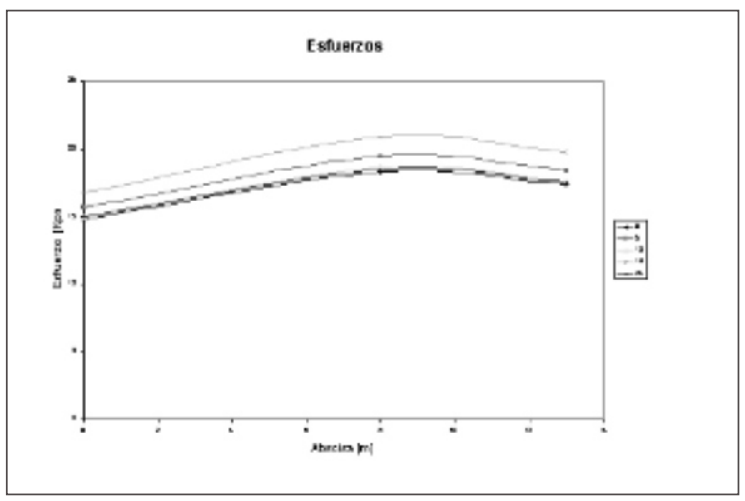

Figura 6. Esfuerzo para un profundidad de $7.5 \mathrm{~m}$.

Para finalizar, en la Figura 7 se indican los resultados obtenidos para el mismo grupo de zapatas, pero a una profundidad de $0.5 \mathrm{~m}$. Se puede apreciar que el efecto de superposición resulta despreciable para pequeñas profundidades. En este sentido, para una profundidad de $0.5 \mathrm{~m}$ el efecto de superposición sólo incrementa el esfuerzo en $0.03 \mathrm{Kpa}$, mientras que para la profundidad de $7.5 \mathrm{~m}$ el incremento máximo fue del orden de $8 \mathrm{Kpa}$.

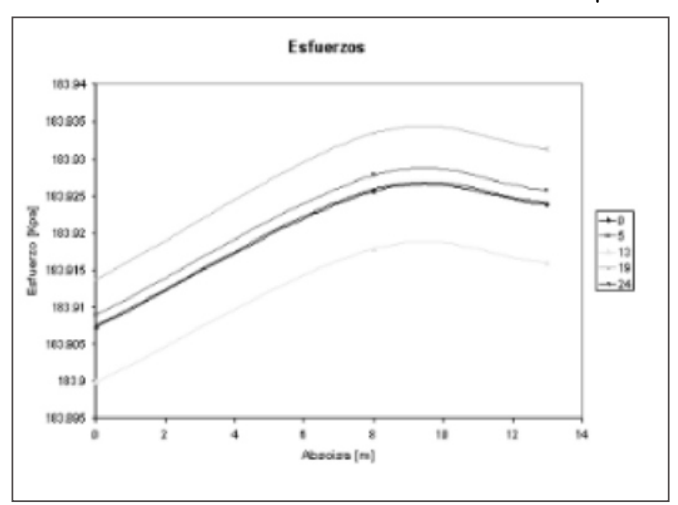

Figura 7. Esfuerzos bajo cada una de las zapatas a una profundidad de $0.5 \mathrm{~m}$.

\section{ASIGNACIÓN DE TAREAS}

En el numeral anterior se consideró una aplicación desarrollada para los cursos de geotecnia impartidos en la Pontificia Universidad Javeriana-Cali. Sin embargo, el desarrollo por parte de los estudiantes de sus propios programas es fundamental en el proceso enseñanza aprendizaje, dado que estimula, entre otros, los siguientes procesos:

1. Activación de conocimiento previo, ya que el aprendizaje no toma lugar de la nada. En su lugar las personas aprenden por asimilación de la información nueva en las estructuras cognoscitivas existentes (Gagne, 1985). En este sentido, la programación permite profundizar en un tema e investigar sus relaciones con sus antecesores.

2. Resalta la relevancia de un tema, dado que ésta, aparentemente, es una condición necesaria para que la mayoría de estudiantes adopten una actitud profunda respecto al aprendizaje (Felder, 1992).

3.Permite clarificar, organizar y resumir el material del curso; esto dado que usualmente el desarrollo de una aplicación implica la revisión de gran parte del material previo.

4. Desarrolla y fortalece la creatividad; dado que, en cada aplicación, el estudiante propone soluciones innovadoras e imaginativas.

A partir de las consideraciones anteriores, a continuación se listan algunas de las tareas asignadas durante los diferentes cursos de geotecnia:

1. Clasificación de suelos. Para tal fin se deben suministrar los resultados de los análisis granulométricos y los limites de consistencia.

2. Cálculo de esfuerzos verticales y laterales.

3. Determinación de parámetros geotécnicos a partir de diferentes correlaciones y metodologías (Gonzales, 1999).

4. Cálculo del factor de seguridad para un talud en suelo homogéneo y una superficie de falla circular. 
5. Determinación del potencial de licuación por métodos empíricos (Youd, et. Al, 1997).

6. Obtención de los espectros elásticos de respuesta para sistemas de un grado de libertad.

\section{CONCLUSIONES}

Se ha resaltado la importancia del uso del computador y en particular de la programación en Visual Basic para Excel, en la enseñanza de ingeniería, presentando una disciplina especifica como la geotecnia, en la cual dada la cantidad de variables e incertidumbres implicadas resulta fundamental el análisis paramétrico.

El desarrollo de software permite: activar el conocimiento previo; resaltar la relevancia de un tema; clarificar, organizar y resumir el material de un curso; desarrollar y fortalecer la creatividad.

Se concluye que ayudas computacionales facilitan de manera considerable el trabajo de aplicación de las teorías y los modelos matemáticos en la solución de los problemas de ingeniería, como es el caso de geotecnia en ingeniería civil, pero se debe hacer claridad que esta herramienta se debe completar con las técnicas de exploración, la experiencia y el criterio del ingeniero. Sin embargo, las simulaciones realizadas permiten una mayor confiabilidad en las decisiones tomadas.

El desarrollo de código para la solución de problemas de ingenieria resulta de gran ayuda en el proceso enseñanza aprendizaje, dado que le permite al estudiante afianzar los diferentes conceptos y lo enfrenta con diversos problemas prácticos.

\section{AGRADECIMIENTOS}

Los autores agradecen a las Universidades Javeriana - Cali y a la Universidad del Valle por el apoyo durante la realización de este trabajo.

\section{BIBLIOGRAFÍA}

Berry, P. L. y Reid, D., 1993. "Mecánica de Suelos", McGraw Hill Interamericana, S.A.

Bowles, J.E., 1996. "Foundation Analysis and Design, 5 th Ed.", Mc Graw Hill Book Co.

Brent, R. y Felder, R., 1992. "Writing Assignments- Pathways to Connections, Clarity, Creativity", College Teaching, 40(2), 43-47 (1992).

Cornell, G., 1994. "Manual de Visual Basic 3 para Windows", McGraw Hill Interamericana de España. Madrid 1994.

Coronado, C. A., 2000. "Cursos de Geotecnia", Pontificia Universidad Javeriana, Sede Cali. http://escer.puj.edu.co/ civil/default.htm, Departamento de Ingeniería Civil: Universidad Javeriana, Santiago de Cali.

Gagne, R.M., 1985. "The Conditions of Learning and Theory of Instruction", 4th Ed., New York: CBS College Publishing.

Gonzales, A., 1999. "Estimativos de Parámetros Efectivos de Resistencia con el SPT". X Jornadas Geotécnicas de la InORMADAS GEOTÉCMICAS DE LA IMGEMIERÍA COLOMBIAMA, GEOTECMIA EM AMBIENTES SISMICOS Y VOLCÁMICOS. Santa Fe de Bogotá, D.C. Moviembre 3, 4 y 5 de 1999.

HEYMAM, Mark S. La esencia de Visual Basic 4. Prentice-Hall Hispanoamericana, S.A. México 1996.

HOLT2, Robert. An introduction to geotechnical engineering. Estados Unidos : Prentice Hall, c1981.

Jacobson, Reed. Programación con Microsoft Excel 2000, Macros y Visual Basic para aplicaciones. McGraw Hill Interamericana de España. Madrid 1999. 
LAMBE, T.W. y WHITMAM, R.V., 1972. "Mecánica de Suelos", Limusa - Wiley, México.

Microsoft Corporation. Office97 / Visual Basic Programer's Guide. ISBM: 1-57231-340-4. Abril 1997. http://msdn.microsoft.com/library/.

Microsoft, EXCEL. 1983-1995 Copyright Microsoft corporation, Todos los derechos revervados.

SHAMK, David. ROBERTS, Mark. MYERS, Tamra. Microsoft Office2000 / Visual Basic Programer's Guide. http://msdn.microsoft.com/library/.

Wankant, P., Oreovicz, F. Teaching engineering. Purdue University.

Youd, T.L and Idriss, I.M, eds., 1997. Proceedings of the MCEER Workshop on Evaluation of Liquefaction Resistance of Soils, Tech. Rep. MCEER-97-0022, Mational Center for Earthquake Engineering Research, Buffalo. 